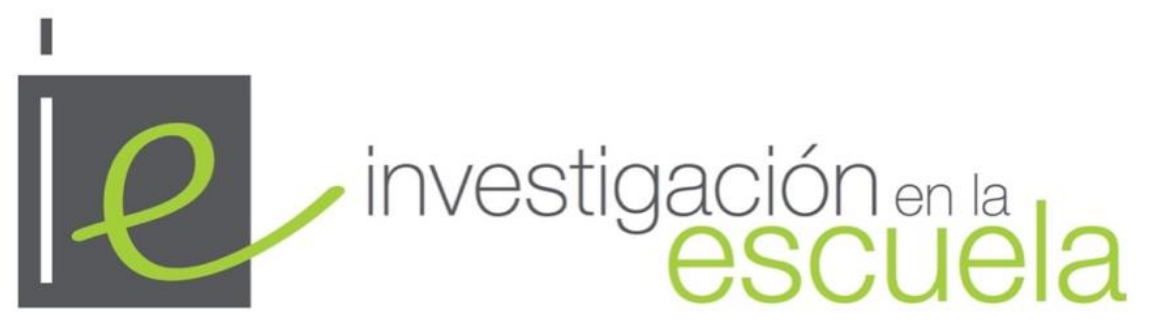

Revista de Investigación e Innovación Educativa n ${ }^{\circ}$ 104, 2021| e-ISSN 2443-9991

\title{
Dificultades y posibilidades para educar en ciudadanía en la Universidad.
}

\author{
Difficulties and possibilities to educate in citizenship at University
}

iD Dr. José Francisco González Puentes es Profesor Titular en la Facultad de Educación de la Universidad El Bosque (Colombia)·fgonzal_99@hotmail.com·https://orcid.org/0000-0001-7024-5941

Cómo citar este artículo

González Puentes, J. F. (2021). Dificultades y posibilidades para educar en ciudadanía en la Universidad. Investigación en la Escuela, 104, 53-64. doi: http://dx.doi.org/10.12795/IE.2021.i104.05

Resumen. El presente artículo analiza las posibilidades y dificultades que se han venido presentado al interior de la universidad para educar en ciudadanía a sus estudiantes. Los análisis se realizan desde una perspectiva de síntesis y de reflexión valorativa, que se sustenta en algunos resultados de investigaciones sobre educación ciudadana y formación del profesorado en los que se ha participado, así como en otras aportaciones recientes de simposios y publicaciones internacionales relacionadas con la educación ciudadana. El propósito es promover la discusión en torno a un tema tan importante para la sociedad en estos tiempos y exponer algunas implicaciones para la mejora de la educación universitaria y, especialmente, de la formación del profesorado.

Abstract. This article analyzes the possibilities and difficulties that have arisen within the university to educate students in citizenship. The analyses are carried out from a perspective of synthesis and valuative reflection, which is based on some research results on citizenship education and teacher training in which we have participated and on other recent contributions from international symposia and publications related to citizenship education. The purpose is to promote discussion around such an important issue for society in these times and to expose some implications for the improvement of university education and, especially, teacher training.

\section{Palabras clave $\cdot$ Keywords}

Educación ciudadana, responsabilidad social universitaria, formación del profesorado, planificación de la educación, currículos, universidad.

Citizen education, university social responsibility, teacher training, education planning, curriculum, university.

\section{Universidad y educación ciudadana}

La institución universitaria tiene una especial responsabilidad en la tarea de la construcción de ciudadanía, por muy diversas razones. De manera directa, porque la universidad forma a un sector importante de la población en los distintos ámbitos profesionales, y, de manera indirecta, porque esos profesionales van a incidir en sectores importantes del funcionamiento de la sociedad.

Esta responsabilidad general la debe ejercer la universidad a través de las tres grandes funciones que le competen: la docencia, la investigación y la extensión o función propiamente social. Como señala Martínez Martín (2006), "una universidad que descuidara o no prestara la atención, sin duda laboriosa y delicada, a las tres funciones citadas no sería a nuestro entender una universidad de calidad” (p. 86). Se puede interpretar más ajustadamente esa función de la universidad desde el concepto de Responsabilidad Social Universitaria, 
un concepto surgido en la pasada década de los noventa y que desde entonces ha sido objeto de atención y debate.

En ese sentido, la UNESCO (1998; 2009; 2016) y otras instituciones internacionales han destacado la especial responsabilidad de la universidad en la interpretación de las complejas situaciones de nuestro mundo y en el aporte de mecanismos para afrontar esas situaciones; más aún en la actual coyuntura mundial, con gravísimos problemas como el agotamiento de recursos básicos, la pérdida de biodiversidad o la incontenible contaminación -con sus más graves consecuencias en el calentamiento global y el consiguiente cambio climático-, problemas que no se habían presentado con estas dimensiones en otros momentos históricos. Por ello, resulta urgente postular un modelo de universidad comprometida con los problemas de la sociedad (Evia Alamilla et al., 2017; Manzano-Arrondo, 2011), lo que, por lo demás, exigiría "nuevas metodologías y formación previa del profesorado en el desarrollo de las competencias para la capacitación socialmente responsable del alumnado" (Martínez-Usarralde et al., 2017, p. 15).

En efecto, en la situación de emergencia que estamos viviendo, la sociedad reclama al sistema educativo que, con visión de futuro, sea capaz de formar ciudadanos para los retos profesionales y laborales venideros, tal como lo reclaman científicos y académicos (Fernández Rey y Fernández Sera, 2010; Lozano Estivalis et al., 2018; Santisteban Fernández, 2012). De hecho, como ya en su momento recordaba Morín (2001), es prioritario educar no para las certezas sino para la incertidumbre. Al respecto, Padilla et al. (2012) afirman que "se necesita una educación que no deje a los ciudadanos inermes frente a las poderosas estratagemas de que hoy disponen los medios masivos para camuflar sus intereses y disfrazarlos de opinión pública" (p. 59); y mucho más en la situación de penetración del modelo educativo neoliberal que ha provocado la remodelación de programas en las universidades y las ha convertido, a pasos acelerados, en "instituciones para el entrenamiento profesional y cada vez menos [en] universidades en el sentido del saber para transformar, hacer ciencia y socializar a favor de una identidad ciudadana crítica" (Mollis, 2010, cit. en GascaPliego y Olvera-García, 2011, p. 39). En este sentido, y en concordancia con García Pérez et al. (2020, p. 55), asumo un modelo de "universidad ciudadana, realmente comprometida con la sociedad, que conjugue de manera compleja las funciones universitarias de educar, investigar y transformar".

Lamentablemente esto no es lo que ocurre; al contrario, el diseño de las carreras académicas se construye tradicionalmente en conexión con el desarrollo de la dimensión investigadora, y se deja en un segundo plano la enseñanza y la extensión. De hecho, esta última función suele quedar reducida a la relación universidad-empresa, sesgada hacia una modalidad de transferencia dirigida al mercado, y desde una óptica tecnocrática que olvida su profunda potencialidad de transformación social. Con demasiada frecuencia, la institución universitaria recela de esa dimensión formativa o bien considera que no le compete porque, de acuerdo con Martínez Martín (2006), "la formación ética dirigida a la construcción de la personalidad moral y a la formación ciudadana no son objetivos que, de forma habitual, puedan identificarse como tales en la concreción de los planes docentes y en las prácticas de aprendizaje en la universidad” (p. 90). En relación con esta responsabilidad de cualquier institución universitaria, se podrían distinguir tres posiciones: 1) "la universidad conoce y potencia la cultura política"; 2) "la universidad está al margen, pero [...] sus docentes tienen libertad de cátedra y lo hacen por iniciativa particular"; y 3) se separa claramente "la formación académica del quehacer político del país" (Martínez Martín, 2006, p. 25).

Con respecto a esta discusión, defiendo la idea de que la universidad debe responder al gran desafío que supone la grave situación del mundo en el siglo XXI -antes esbozada-, frente a la concepción de la educación como un mero producto del mercado, como un bien de consumo que puede ser comprado por el consumidor individual o apropiado por las grandes corporaciones. Tal como afirma Salazar (2003):

El sentido social de la educación universitaria está dado por su contribución efectiva a la formación de mejores ciudadanos profesionales, conscientes de sí y de los demás, partícipes en la preservación de la salud personal y ambiental, en su desarrollo y en el de la sociedad en la que se desenvuelven, es decir, se trata de formar ciudadanos capaces de ejercer su profesión con sentido de responsabilidad por la calidad de vida de sí mismos y de la colectividad. El reto es el de construir ciudadanía en la formación integral de profesionales (p. 32).

En ese sentido, no debería separarse la vida académica de la vida política (entendida en su acepción más básica), dado que, en la universidad, de la mano de la formación profesional, debe desarrollarse una sólida formación en ciudadanía, pues la formación en valores democráticos tiene que ser inherente a la educación en todas sus etapas, y con mayor razón en la formación profesional. A este respecto, la educación superior tiene la responsabilidad de "elevar los conocimientos y los planteamientos humanos y sociales al nivel de adulto, la formación profesional necesaria y conducente a la primera inserción laboral de las personas y a su consiguiente autonomía económica y social, y la formación para el ejercicio de la ciudadanía adulta (Cobo Suero, 2003, p. 360). 
Por ello, la universidad tiene, en definitiva, la función de formar profesionales competentes, con un sentido ético de la profesión y un sentido social de contribución cívica a la sociedad (Cobo Suero, 2003). Por lo demás, la etapa de formación universitaria es especialmente indicada para la socialización política en una edad ya adulta y en un contexto formativo propicio para ello (López López, 2008). Dado que la ciudadanía se aprende, sobre todo, en la experiencia vital y en la participación en los ámbitos en los que se desarrolla la actividad cotidiana del individuo, el contexto universitario resulta, en principio, especialmente indicado para asumir la ciudadanía como cultura. En ese sentido, "la universidad es una institución que enseña pero que también aprende para transformarse a sí misma y poder intervenir en la transformación y mejora de la realidad" (Ramalho y Beltrán, 2012, p. 35), en una sinergia de reflexión-acción. Y ello es indispensable, con mayor razón, en el caso de la formación de los profesionales que se van a dedicar a la docencia (Albalá-Genol et al., 2019).

Pero, como he señalado antes, en la universidad no hay cultura de participación. Es frecuente que hoy en el mundo universitario haya una baja participación del estudiantado en la gestión universitaria e, incluso, en las actividades culturales. Esto es

una realidad generalizada en las universidades españolas. Pese a algunas acciones para potenciarla, todavía no se ha extendido a una cultura de participación activa en la que el alumnado se integre plenamente en la gestión y vida universitaria, más allá de lo académico (Auar, 2011, p. 1).

Este fenómeno sucede porque la motivación principal suele ser la finalización de la carrera; ello se explicaría, sobre todo, por la extensión de la concepción dominante del estudiante (y de su familia) como "cliente" que compra el servicio de la universidad como una mercancía, como una mera herramienta de preparación para encontrar un buen puesto laboral (Vallaeys y Carrizo, 2006).

Sin embargo, la verdadera participación implica información y formación adecuadas, de modo que el estudiante pueda

conocer qué opciones de participación posee y cuáles son las vías para llegar a ellas eficazmente; si el estudiantado desconoce, de un lado, que es posible participar y, en segundo lugar, cómo llevar a cabo sus motivaciones de participación, no existirá implicación (Auar, 2011, p. 4).

Esta participación es "responsabilidad de todos los sectores -estudiantado, gestores, docentes, políticos- y beneficia tanto al alumnado como a la propia universidad" (Auar, 2011, p. 8). En todo caso, deberíamos considerarla como un proceso de construcción, que podría ir desde experiencias más guiadas y centradas en la información hasta experiencias más complejas, reflexivas y comprometidas con el cambio social (Hart, 2001; Kerr, 2000).

Establecida la necesidad y la exigencia de una formación ciudadana como función ineludible de la universidad, es necesario explicitar qué tipo de ciudadanía se toma como referencia para dicha formación. En coherencia con los rasgos que he venido apuntando, se trataría de una ciudadanía claramente activa y participativa, comprometida con los problemas sociales y ambientales y, por tanto, con un carácter planetario, que implicaría la intervención tanto en la escala local como en la global, y que incluya, entre otros aspectos, la educación política y, lógicamente, la educación para la democracia, además de la perspectiva ética (García Pérez y De Alba Fernández, 2011).

En efecto, este tipo de ciudadanía activa y comprometida es tanto más necesaria en un mundo que, como antes se señaló, tiene un sinnúmero de problemáticas por atender y en el que la comprensión de lo que ocurre y la intervención activa en relación con ello exige una perspectiva claramente global (Barrow, 2017; García Pérez, 2019; Goren y Yemini, 2017; Henderson y Wright, 2015). Así lo expresan otras instituciones internacionales, como la propia UNESCO (2016), que afirma que en un mundo globalizado se requiere una

pedagogía transformadora que permita a los alumnos resolver problemas persistentes relacionados con

el desarrollo sostenible y la paz, que conciernen a toda la humanidad y los equipe con los conocimientos, competencias, actitudes y comportamientos que se necesitan para ser ciudadanos informados, comprometidos y solidarios (p. 12).

En ese tipo de ciudadanía habría que formar a la totalidad del estudiantado en el ámbito universitario. Por ende, para desarrollar una educación en un modelo de ciudadanía como el que he venido caracterizando, tiene que haber profesores y profesoras capacitados para desarrollar esa función: la formación de ciudadanas y ciudadanos críticos, reflexivos, participativos, comprometidos con la solución de problemas. Sin embargo, esta función no se cumple adecuadamente, como reflejan diversas investigaciones (Estellés y Romero, 2018; Molina et al., 2013). Por lo que resulta tanto más necesaria la intervención en este ámbito, una intervención de debe tener sus fundamentos en la investigación. 


\section{La investigación sobre educación ciudadana y formación del profesorado}

Sin pretensión de ofrecer una revisión general sobre la investigación en torno a la educación ciudadana en la universidad, y más concretamente en la formación del futuro profesorado, voy a presentar, en los apartados siguientes de este artículo, algunos resultados de investigaciones sobre ese campo, con el propósito de promover la reflexión en torno al mismo y exponer algunas implicaciones para la mejora de la educación y, especialmente de la formación del profesorado. Me basaré para ello en un conjunto de investigaciones de la última década que conozco más de cerca por haber estado implicado en las mismas o haber tenido contacto próximo con ellas. Las relacionaré, en todo caso, con otras investigaciones similares recientes.

He tomado como punto inicial de referencia los resultados de tres proyectos de investigación que han abordado situaciones de educación para la ciudadanía y formación del profesorado en España, algunos países europeos (Portugal, Italia, Francia) y algunos países latinoamericanos (Colombia, Argentina, Brasil). El primero fue el proyecto I $+\mathrm{D}+\mathrm{i}$ Estrategias de formación del profesorado para educar en la participación ciudadana (García Pérez, 2011), desarrollado en los años 2012 a 2014, que se apoyó, a su vez, sobre las conclusiones de un proyecto anterior, Educación para la ciudadanía y formación del profesorado: dificultades y posibilidades para educar en la participación ciudadana (García Pérez, 2006), desarrollado entre 2006 y 2009. Los resultados de dicha investigación fueron dados a conocer en diversas publicaciones, siendo destacable el elenco de las mismas que quedó recogido en las actas de un simposio internacional dedicado específicamente a la educación para la participación ciudadana (De Alba Fernández et al., 2012).

El segundo proyecto fue un proyecto Erasmus Plus, Pedagogia della cittadinanza e formazione degli insegnanti: un'alleanza tra scuola e territorio, en siglas STEP: School, Territory, Environment, Pedagogy (Nigris, 2015), desarrollado entre 2015 y 2018. Las principales conclusiones de este proyecto europeo se recogieron en la publicación compilatoria Novi Cives. Cittadini dall'infanzia in poi (Borghi et al., 2015), habiéndose publicado también otros resultados parciales, como es el caso de los referidos a la universidad de Sevilla en España y a la universidad de Ijuí (Unijuí) en Brasil (Callai y De Moraes, 2016).

$\mathrm{El}$ tercer proyecto surge como consecuencia de las anteriores investigaciones, fue financiado por la Universidad El Bosque de Colombia y su título es Educación para la ciudadanía: dificultades y posibilidades de los currículos y los docentes de la Universidad El Bosque para educar en la participación ciudadana. Se desarrolló entre el 2015 y el 2018 (González Puentes, 2015). Sus resultados se han venido presentado en diversos congresos y publicaciones institucionales.

En esa línea, he prestado también atención a resultados de investigación recogidos en los últimos años en el ámbito iberoamericano, como es el caso de los simposios internacionales organizados anualmente por la Asociación Universitaria de Profesorado de Didáctica de las Ciencias Sociales, de España; así el dedicado en 2019 a formación del profesorado (Hortas et al., 2019), con abundantes aportaciones relativas a la formación inicial de docentes para ser educadores en ciudadanía, o la obra de compilación de investigaciones y experiencias de participación ciudadana en el contexto escolar (Claudino et al., 2019), que recoge conclusiones de un congreso específico celebrado en Lisboa, también en 2019. Asimismo, han aparecido en los últimos años algunas compilaciones que he considerado de específico interés, como es el caso del handbook que recoge aportaciones investigadoras de Europa y Latinoamérica (y en menor medida de otras zonas del mundo) sobre esta temática (Pineda-Alfonso et al., 2018), así como otras de carácter similar (Gomes Dias et. al, 2020).

Por lo demás, se han consultado artículos diversos que, a su vez, habían revisado la producción de investigaciones recientes sobre educación para la ciudadanía y formación del profesorado, con especial atención al contexto iberoamericano (por ejemplo, Arroyo Mora et al., 2020; Garcés, 2020, 2021; Salinas Valdés et al., 2016).

A partir de este recorrido exploratorio -que toma como punto de partida los proyectos citados- y contrastando resultados con otras aportaciones (que se irán presentando a modo de discusión), he allegado algunos resultados que considero relevantes y que presento a continuación, como síntesis reflexiva, con propuestas para la mejora de la formación del profesorado, que tiene, en definitiva, la responsabilidad de educar para la ciudadanía en el difícil contexto mundial actual de problemáticas sociales y ambientales.

\section{Algunos resultados e implicaciones para la educación}

En el marco de las anteriores consideraciones, presento a continuación, a modo de reflexión y discusión, una selección de los resultados extraídos, desde la perspectiva de las dificultades y posibilidades para formar en ciudadanía participativa al estudiantado universitario y en especial a los futuros profesionales 
de la docencia. Lo haré atendiendo a diversos aspectos y actores: los currículos, el profesorado, los estudiantes y la cultura institucional universitaria.

\subsection{Currículos}

En términos generales, se puede afirmar que los currículos universitarios hacen muy poca referencia a educación ciudadana y, cuando la consideran, se suelen quedar en el plano de las buenas intenciones, o bien la consideran responsabilidad de determinadas asignaturas o actividades complementarias, que asumirían por sí solas la función de formar integralmente a los estudiantes universitarios como ciudadanos. Con relación a las asignaturas que integran esta perspectiva, la mayoría de ellas se encuentran en el ámbito de las ciencias sociales o humanas. En algunos casos, se ofrecen asignaturas con esta temática específica como materias optativas o electivas, a las que algunos estudiantes optan libremente, y tienen el propósito de presentar, desde una mirada puramente disciplinar, contenidos filosóficos, sociológicos o jurídicos sobre la ciudadanía o temas relacionados como la participación, la democracia, etc. (González Puentes et al., 2016). A este respecto, habría que tener en cuenta que la enseñanza de la ciudadanía requiere, como soporte, contenidos disciplinares, pero ello no se puede dejar a merced de una asignatura desconectada de las demás del currículo y con poca incidencia en la formación integral de los universitarios, en especial cuando se trata de los futuros profesores.

Por lo demás, los escasos contenidos relacionados con la ciudadanía que se formulan en la mayoría de los currículos están más bien relacionados con el conocimiento de datos, hechos, conceptos sencillos, pero, en pocas ocasiones, con conceptos complejos o con principios y teorías (aunque sean sencillas). Son escasos los que se refieren a la ejecución de procedimientos, estrategias, técnicas, habilidades, destrezas, métodos, etc., (contenidos procedimentales), así como a contenidos relacionados con los valores y las actitudes; un aspecto corroborado por diversas investigaciones, como la de Flores-González y García-González (2014), quienes señalan que:

[...] el currículo ha continuado enfatizando la dimensión política de la ciudadanía con contenidos centrados en la institucionalidad política y en el incentivo de una participación responsable y representativa y ha dejado en un segundo plano las dimensiones sociales y civiles de la ciudadanía y el desarrollo de la capacidad crítica que requiere la participación activa (p. 41).

Se podría deducir entonces que las asignaturas relacionadas con la educación para la ciudadanía tienen mayormente el propósito de informar, pero no de afectar a otras dimensiones de los profesores en formación para que puedan encontrar las motivaciones, los procedimientos, las actitudes y los valores necesarios para participar. Lo que agrava este panorama es que en muchos de los casos los contenidos sobre educación ciudadana que se postulan en los currículos y en los syllabus de las carreras no se desarrollan de forma concreta en las aulas universitarias. Es decir, a medida que se va descendiendo desde las declaraciones más generales hasta los materiales didácticos y las actividades de aula, los buenos propósitos iniciales se van diluyendo En consecuencia, "la construcción de ciudadanía corre el riesgo de ser una buena intención mal implantada, reducida a contenido añadido como parche, sin objetivos claros" (Salazar, 2003, p. 41).

Con relación a las actividades complementarias de los currículos, con frecuencia las universidades programan eventos como foros, encuentros, cátedras abiertas o congresos, con el propósito de brindar a sus estudiantes un espacio para el aprendizaje de la ciudadanía. La intención es loable y representa una oportunidad, pero no es suficiente, ya que estos espacios académicos son aprovechados por un número reducido de profesores y estudiantes, y la gran mayoría de los integrantes de la comunidad educativa no los valoran como verdaderos espacios de formación. La educación para la ciudadanía no puede quedar reducida a actividades desarticuladas de los currículos ni mucho menos al espacio curricular de una asignatura.

$\mathrm{Al}$ respecto, la universidad asume el reto de formar a sus estudiantes con las más altas calidades científicas, pedagógicas y técnicas, pero también tiene la responsabilidad de implementar estrategias de gestión curricular que garanticen la formación de ciudadanos "capaces de ejercer su profesión con sentido de responsabilidad por la calidad de vida de sí mismos y de la colectividad. El reto es el de construir ciudadanía en la formación integral de profesionales" (Salazar, 2003, p. 32), pues "la construcción de ciudadanía en el currículo requiere del compromiso de las autoridades académicas para que haya consistencia entre el currículo explícito en los papeles y el currículo implícito en las acciones" (p. 39). En definitiva, se requieren cambios importantes en los currículos para que las ciudadanas y ciudadanos -y en especial quienes van a ser formadores de ciudadanía- puedan afrontar con la preparación y responsabilidad necesarias sus profesiones, desarrollando, como señalan Holguín Villa et al. (2019, p. 89), capacidades relacionadas con "la toma de decisiones por sí mismos, teniendo conciencia crítica sobre las mismas, asumiéndose como ser ciudadanos del mundo, reconociendo al otro en sus necesidades y problemáticas". 


\subsection{Profesorado}

Las dificultades de los profesores y profesoras para educar en ciudadanía a sus estudiantes podrían estar asociadas a las concepciones que aquellos poseen sobre ciudadanía y educación para la ciudadanía en la universidad, al modelo pedagógico que orienta su práctica docente y a la falta de formación. Gran parte de los profesores entienden la ciudadanía en términos de pertenencia a una comunidad u ordenamiento social y político, geográficamente determinado por los límites de un país o Estado, como destaca concretamente el estudio de Salinas Valdés et al. (2016). Esta posición se corresponde con una concepción legalista, que considera que la ciudadanía supone un determinado estatus social, con sus derechos y deberes. En consecuencia, se presenta una dificultad ideológica para educar en ciudadanía, ya que los docentes no llegan a concebir esa educación como un proceso y una tarea por construir con un carácter dinámico, cambiante y orgánico; no la entienden como un proceso, sino como un resultado. Una concepción activa de ciudadanía, como se señaló al comienzo de este artículo, implica compromiso social y participación activa en relación con los problemas sociales y ambientales; por tanto, exige una perspectiva inevitablemente global (García Pérez, 2019, 2021: García Pérez et al., 2020).

Como resultado, el profesorado universitario considera que la educación ciudadana en la universidad no forma parte de sus deberes, que los estudiantes llegan a la universidad suficientemente formados en ello desde la educación primaria y secundaria, que la ciudadanía es un problema de otras asignaturas y de otros docentes, que los responsables de esta tarea son la familia u otros estamentos de la sociedad. Asimismo, confirmando lo dicho más arriba, suelen considerar que los currículos universitarios solo tienen espacio para las asignaturas propias del campo disciplinar correspondiente, debiéndose dedicar los docentes a formar en las competencias específicas relacionadas con los conocimientos científicos, tecnológicos y con los aspectos laborales o profesionales relacionados con la futura profesión (González Puentes, 2013).

Pero desconocer, por una u otra razón, la importancia de la educación ciudadana en la formación universitaria no puede ser una justificación o excusa para que los docentes universitarios no se comprometan activamente en la formación de sus estudiantes. El profesorado universitario, independientemente del área de conocimiento a la que pertenezca, tiene la responsabilidad ética de contribuir a la formación ciudadana desde sus aulas. Para que esto ocurra se necesita superar viejas posturas "mediante la sensibilización, la información y la formación de los profesores universitarios" (Salazar, 2003 p. 39), y, además, que los docentes quieran cambiar y asumir esta responsabilidad: nada cambiará, así lo postulen las políticas y los currículos oficiales universitarios. Por ello, "por razones de ética profesional e institucional, cabe exigir a los docentes que se impliquen activamente en la vida, la deliberación, las decisiones y el proyecto pedagógico de la institución donde trabajan" (Muñoz Zamora, 2011 p. 101).

Como factor de fondo, el modelo pedagógico imperante en la docencia universitaria se basa en la transmisión de contenidos, y esto se constituye en un obstáculo porque no favorece la generación de espacios educativos apropiados para el aprendizaje de la ciudadanía, ya que, como señalan De Alba Fernández y Porlán Ariza (2017):

Todo el protagonismo corresponde al docente que, como experto en el conocimiento que se pretende enseñar, intenta hacerlo llegar al estudiante a través de la explicación. El papel de estos se limita a intentar captar la información emitida del modo más fiel posible. En este modelo el conocimiento se concibe como una verdad acabada. Desde el punto de vista psicológico, se asume que el conocimiento es algo externo al que aprende y que puede ser adquirido gracias al proceso de transición. En este sentido, la mente del estudiante sería como un recipiente vacío que debe ser rellenado por el profesor. (p. 38)

Como se aprecia, el modelo pedagógico transmisivo no genera espacios educativos que promuevan los aprendizajes que se requieren para que los estudiantes y los profesores puedan participar activamente en la vida universitaria. Construir democracia y participación en la universidad requiere, por una parte, de espacios de reflexión crítica en los que los diferentes actores educativos "aprendan el valor del diálogo, enmarcado en valores como el respeto, la confianza, la diversidad, para construir desde la práctica una ética ciudadana" (Muñoz Zamora, 2011, p. 123), y, por otra, concebir la educación ciudadana como "un proceso de pedagogía democrática en el que las personas toman conciencia de su condición como sujetos de decisiones y van adquiriendo una formación ciudadana enfocada hacia la práctica" (Velásquez, 2003, p. 119). Esto en aras de que la acción docente se comprenda como una perspectiva de desarrollo humano y un medio para la convivencia social y el ejercicio de la ciudadanía (Magendzo, 2004). En ese sentido, "el papel del docente en la construcción de ciudadanía participativa y crítica no es posible sin el diálogo, sin la discusión fundamentada y la participación activa de los estudiantes y los docentes en la experiencia educativa" (Rivera de Parada, 2013, p. 21). 
En concordancia con las investigaciones de González Rivero (2019), es posible afirmar que las profesoras y profesores universitarios son conscientes de la poca preparación que poseen para enfrentar la formación ciudadana de sus estudiantes, pero, también, que esta situación representa una oportunidad de mejora que les permitiría seguir aportando a la construcción de la sociedad y del mundo, como lo han venido haciendo a lo largo de la historia de la universidad.

\subsection{Estudiantes}

En general los estudiantes universitarios piensan que la educación ciudadana no es un contenido importante de su formación profesional y que las actividades programadas por la universidad en ese ámbito no tienen gran relevancia. En definitiva, no valoran este tipo de contenidos porque creen que son añadidos a los contenidos oficiales de los currículos y no tienen gran utilidad a la hora de desempeñarse profesionalmente. Esta concepción se podría atribuir al desconocimiento de los derechos, mecanismos e instancias de participación ciudadana, a una supuesta "falta de madurez" de dichos estudiantes para afrontar y asumir los compromisos y responsabilidades que exigen los procesos de participación ciudadana y, además, a la desinformación sobre los canales y espacios de participación que ofrecen la universidad y las demás instancias del Estado.

Sobre los espacios formales de participación con los que cuenta la universidad, como sus representaciones institucionales, los concejos estudiantiles, los comités de carrera, etc., se detecta que el estudiantado no encuentra grandes motivaciones para participar en dichos espacios porque sienten que son, en gran parte, mecanismos que, en el fondo, no tienen poder real de decisión. La poca credibilidad de los espacios de participación representa una importante dificultad, ya que es allí donde la participación ciudadana se valida como "una estrategia social y política donde el ciudadano ejercen su poder en las decisiones que afecten a su propio bienestar y el de la sociedad, sustentado en valores como la solidaridad, la autonomía y el reconocimiento de la diferencia" (Magendzo, 2004, p. 43).

Inevitablemente, si estos espacios se convierten en ejercicios de participación en los que los estudiantes juegan un papel meramente decorativo, figurativo o de fachada para justificar planes y proyectos de las instituciones universitarias, se estará ayudando a consolidar la cultura de la indiferencia (Hart, 2001). Entonces, se requiere una formación alternativa a la que tradicionalmente se viene impartiendo en la universidad para que "los estudiantes promuevan conductas y convicciones transformadoras sobre los propios supuestos que fundamentan su quehacer en la universidad para que estén en condiciones de participar en la construcción de ciudadanía" (UNESCO, 2016, p. 23).

Por consiguiente, una clave importante de la formación ciudadana de los universitarios y universitarias reside en que realmente practiquen los rasgos básicos de la ciudadanía, como la participación real y comprometida en los asuntos que les afectan, y no que solo sean informados acerca de esas cuestiones. De ahí la importancia de que el diseño y el desarrollo de las asignaturas -en especial en las carreras de formación de docentes- respondan a un modelo didáctico realmente activo (García Pérez, 2017), conforme al cual el aula se convierta en un espacio para trabajar de manera crítica problemas reales, para que los estudiantes sientan que son escuchados, que sus propuestas son valoradas y que estos aprendizajes son útiles "para tomar decisiones fundamentadas y emprender cambios a nivel personal y colectivo, y a escala local y global" (García Pérez et al., 2020, p. 60). Ello no nos puede hacer olvidar las dificultades que esto implica en un contexto escolar (que incluye el contexto universitario), que, por sus características (estructura de espacios y tiempos, carácter cerrado de sus currículos, tradición disciplinar, desconexión del entorno social, etc.), no favorece el ejercicio real de la participación en el ámbito social próximo (García Pérez, 2019).

\subsection{Cultura académica y contexto universitario}

Las dificultades para educar en la participación ciudadana encontradas en los currículos, los docentes y los estudiantes universitarios (especialmente los futuros profesores) guardan estrecha relación y dependencia con la cultura institucional dominante y el contexto en el que se encuentra inmersa la universidad. En ese sentido, la cultura institucional se entiende como

aquella cualidad relativamente estable que resulta de las políticas que afectan a esa institución y de las prácticas de los miembros de un establecimiento. Es el modo en que ambas son percibidas por estos últimos, dando un marco de referencia para la comprensión de las situaciones cotidianas, orientando e influenciando las decisiones y actividades de todos aquellos que actúan en ella (Frigerio et. al, 1992, p. 1). 
Esta genera e impone cierto tipo de pensamiento que acaba reproduciendo rutinas que configuran la identidad de la institución. Aquí, las dificultades estarían relacionadas, por una parte, con la desvinculación del contexto universitario con respecto al contexto social, lo que, según García Pérez (2021), "no propicia una educación cívica activa y participativa" (p. 46), y por otra, con algunos rasgos característicos de la cultura universitaria dominante, rasgos que se ven reflejados en las conductas y comportamientos de los miembros de la comunidad educativa, que se refuerzan y legitiman en la vida diaria y que afectan a todos los actores educativos por igual. Así, por ejemplo, la resistencia al cambio se aprecia en la construcción del currículo cuando los responsables de tan importante tarea piensan que todo está bien y que nada se debe cambiar, o cuando los docentes, a pesar de los procesos de formación pedagógica, siguen enseñando de la misma forma que la que utilizaron sus profesores con ellos.

Al respecto, las aulas "son a menudo decididamente antidemocráticas ya que los profesores con frecuencia ignoran mayormente sus implicaciones y de esa manera perpetúan los comportamientos o creencias no transformativos" (UNESCO, 2016, p. 17), y, en consecuencia, los estudiantes asumen un rol pasivo en el que la participación en el aula y en los demás espacios de la vida universitaria es escasa, donde predomina la cultura del mínimo esfuerzo, que hace que solo se establezcan compromisos con las actividades que conllevan una calificación o un beneficio académico. Por ello, Martínez Martín (2006) concluye que:

no podemos olvidar que una parte importante de las resistencias ante los cambios en la universidad tienen su causa en una actitud pasiva y excesivamente interesada del estudiante en obtener un título que le permita competir con otros en cuestiones estrictamente profesionales y a menudo alejadas o incluso contrarias a propuestas éticas y relativas al mundo de los valores. (p. 88)

Por otra parte, existen otras actitudes y sentimientos propios de la cultura institucional universitaria que hacen que las personas no quieran participar, como, por ejemplo, la crítica destructiva, el miedo a meterse en problemas, el poco respeto por el otro y el desconocimiento de los demás miembros de la comunidad educativa; además, el convencimiento generalizado de que los espacios de participación (a los que la gran mayoría asiste por obligación) son un formalismo que incide muy poco en las dinámicas y decisiones de la universidad. Esto evidencia que se necesita con urgencia "fortalecer la cultura política democrática en las universidades" (Rivera de Parada, 2013, p. 24). Dicho propósito se podría alcanzar si la cultura institucional se entendiera como un ámbito de aprendizaje de la ética en la que todos sus integrantes aprenden y conviven. En definitiva, "una cultura de la comunidad en la que participan todos y en la que se pueden ejercer y reclamar sus derechos, además de participar en la toma de decisiones que afecta al conjunto" (Martínez Martín, 2006, p. 95).

En estrecha relación con lo anterior, el contexto en el que se encuentra inmersa la universidad también incide en la configuración de las dificultades para educar en ciudadanía. En términos generales, los graves problemas que afectan a las sociedades de todo el mundo, como, por ejemplo, la corrupción y el deterioro de la política, hacen que los ciudadanos sientan apatía, desconfianza y desinterés por la participación ciudadana y crean que no sirve para nada y que es un problema de otros. El panorama se complica si se tiene en cuenta que un número importante de personas no tienen una formación política y, además, los pocos conocimientos con los que cuentan se hallan influenciados por los medios de comunicación que manipulan la opinión de la ciudadanía. En este sentido, la universidad "tiene que adaptarse a su entorno y, a su vez, adaptar el entorno a sus propios principios y valores. Es totalmente legítimo pensar que la universidad puede influir e, incluso, formar el juicio ético-ciudadano de sus miembros" (Gasca-Pliego y Olvera-Garcia, 2011, p. 51).

A pesar de las dificultades mencionadas y de las conclusiones de algunas investigaciones que afirman que la universidad, en la actualidad, no se concibe como espacio de formación ciudadana, sino como espacio de aprendizaje de lo académico y laboral (González Puentes, 2013), la universidad "no puede eludir el deber formativo político y democrático. Las disciplinas y los conocimientos que imparte quedarían limitados sin esta dimensión política y sin esta proyección social del saber” (Soler et al., 2012, p. 546).

\section{Consideraciones finales}

Indudablemente, la formación ciudadana de los estudiantes debería ser ejercida por la institución universitaria de manera interdisciplinaria, de modo que se proporcione un contexto formativo general como ya se ha indicado- con un currículo adecuado que asuma esta dimensión a través de "proyectos y programas incorporados a diferentes áreas del conocimiento de todos los programas universitarios que propicien acciones dentro y fuera de las instituciones de educación, con diferente tipo de ofertas para la población según las potencialidades y sus dinámicas" (Holguín Villa et al., 2019, p. 83). En ese sentido, 
la formación ciudadana es un reto experiencial y de vida para cada dependencia y cada lugar del campus universitario con todos los integrantes de la comunidad, de suerte que sea promovida una actitud de conciencia de sí y de vinculación con los otros y con el entorno; un conocimiento del tipo de lazos generados con la naturaleza y del tipo de relación con el ecosistema como integrante del mismo. Es vivir la formación ciudadana en los diseños de los planes de desarrollo y programas académicos continuamente realimentada y dinamizada por las experiencias y vivencias en las interacciones cotidianas (Holguín Villa et al., 2019, p. 84).

Para que la universidad pueda afrontar este reto, debe tomar importantes decisiones sobre los supuestos filosóficos y pedagógicos orientados a la construcción de los currículos y, al mismo tiempo, implementar programas de formación del profesorado para que esté en condiciones de integrar dicho contenido a su práctica docente, al interior del aula de clase. Esto implica una

revolución educativa, es decir, cambios en las funciones, tanto de las instituciones educativas como en los docentes, que tienen un papel protagónico como actores generadores del cambio en las instituciones de educación superior, por lo que es necesario generar un proceso de formación no solo disciplinar, sino también pedagógica (Moscoso Merchán y Hernández Díaz, 2015, p. 141).

Para ello, el modelo formativo, en concordancia con el marco teórico que he venido exponiendo, debe desarrollar la formación docente según un "modelo investigativo", teniendo como centro el trabajo de diseño y desarrollo de propuestas didácticas centradas, precisamente, en el tratamiento de problemáticas sociales y ambientales que tengan como meta el modelo de ciudadanía propuesto a lo largo de esta reflexión (García Pérez, 2017; Pagès Blanch y Santisteban Fernández, 2011; Pineda-Alfonso, 2015). El marco general en el que se desarrollarían estas actividades seguiría el modelo de trabajo sobre "problemas prácticos profesionales", planteado y experimentado en el Proyecto IRES (García Pérez y Porlán, 2000), un modelo formativo avalado por diversos resultados de investigación (García Pérez et al., 2015).

\section{Referencias}

Albalá-Genol, M. A., Maldonado-Rico, A. F., Etchezahar, E. y Ungaretti, J. (2019). Ciudadanía y participación sociopolítica del futuro profesorado en Madrid (España). Revista Internacional de Investigación en Ciencias Sociales, 15(2), 219-239. DOI: 10.18004/riics.2019.diciembre.219-239. http://revistacientifica.uaa.edu.py/index.php/riics/article/view/829

Arroyo Mora, E., Crespo Torres, B., Mancha Castro, C. y Schugurensky, D. (2020). Prácticas innovadoras en educación ciudadana. ¿Qué dicen las revistas académicas españolas? Revista Fuentes, 22(2), 212-223.

Auar, R. (2011). Las Claves de la participación estudiantil en la universidad española. [Conferencia]. Universidad de Girona. Recuperado de https://dugidoc.udg.edu $/$ bitstream $/$ handle $/ 10256 / 3812 / 155$.pdf?sequence $=1 \&$ is Allowed $=\mathrm{y}$

Barrow, E. (2017). No Global Citizenship? Re-envisioning Global Citizenship Education in Times of Growing Nationalism. The High School Journal, 100(3), 163-165.

Borghi, B., García-Pérez, F. F. y Moreno-Fernández, O. (Eds.). (2015). Novi Cives. Cittadini dallinfanzia in poi. Pàtron Editore.

Callai, H. C. y De Moraes, M. M. (2016). Pesquisa, educação e cidadania. Percursos teóricos e metodológicos. Editora Unijuí.

Claudino, S. et al. (Orgs.) (2019). Geografia, Educação e Cidadania. ZOE/Centro de Estudos Geográficos, Instituto de Geografia e Ordenamento do Território da Universidade de Lisboa. Recuperado de http://www.nosotrosproponemos.com/wpcontent/uploads/2019/06/S_Claudino_et_al_org_2019_Geografia.pdf

Cobo Suero, J. M. (2003). Formación universitaria y educación para la ciudadanía. Revista de Educación, 1, 359-375. Recuperado de https://dialnet.unirioja.es/servlet/articulo?codigo $=761230$

De Alba Fernández, N., García Pérez, F. F. y Santisteban Fernández, A. (Eds.) (2012). Educar para la participación ciudadana en la enseñanza de las ciencias sociales. Díada Editora y Asociación Universitaria de Profesorado de Didáctica de las Ciencias Sociales. Vols. I y II. Recuperado de http://dialnet.unirioja.es/servlet/libro?codigo $=500467$

De Alba Fernández, N. y Porlán Ariza, R. (2017) La metotodolgia de la enseñanza. En R. Porlán (Coord.), Enseñanza universitaria: cómo mejorarla (pp. 37-54). Ediciones Morata.

Estellés, M. y Romero, J. (2018). Tacit assumptions of citizenship education: A case study in Spanish initial teacher education. Education. Citizenship and Social Justice. First Published May 21, 2018. https://doi.org/10.1177/1746197918771336 
Evia Alamilla, N. M., Echeverría Echeverría, R., Carrillo Trujillo, C. D. y Quintal López, R. (2017). Ciudadanía en el currículo educativo: Un análisis del Modelo de Responsabilidad Social Universitaria en una universidad pública. CS, 23, 77-104. http://dx.doi.org/10.18046/recs.i23.2377

Fernández Rey, A. A. y Fernández Sera, A. (2010). La formación ciudadana de los docentes en formación inicial: una prioridad de la universalización de la educación superior pedagógica. Cuadernos de Educación y Desarrollo, 2(17). https://ideas.repec.org/a/erv/cedced/y2010i1726.html

Flores-González, L. M., y García-González, C. A. (2014). Paradojas de la participación juvenil y desafíos de la educación ciudadana en Chile. Magis, Revista Internacional de Investigación en Educación, 6(13), 31-48. https://doi.org/10.11144/Javeriana.M6-13.PPJD

Frigerio, G., Poggi, M. y Tiramonti, G. (1992). La institución educativa. Cara y Ceca. Editorial Troquel.

Garcés, V. H. (2020). Alfabetizar en convivencia ciudadana. Una revisión documental de la educación ciudadana para la resolución de la violencia y el conflicto sociocultural. Sophia, 16(1), 4-18. https://doi.org/10.18634/sophiaj.16v.1i.874

Garcés, V. H. (2021). Educación ciudadana y convivencia democrática. Entre las políticas educativas, la alfabetización escolar y el aprendizaje en la ciudad. Folios, 53, 19-30. https://doi.org/10.17227/folios.53-12906

García Pérez, F. F. (Dir.) (2006). Educación para la ciudadanía y formación del profesorado: dificultades y posibilidades para educar en la participación ciudadana. Ministerio de Educación y Ciencia. España. https://investigacion.us.es/sisius/sis proyecto.php?idproy $=10638$

García Pérez, F. F. (Dir.) (2011). Estrategias de formación del profesorado para educar en la participación ciudadana. Proyecto I+D+i EDU2011-23213. Ministerio de Ciencia e Innovación. España. https://investigacion.us.es/sisius/sis proyecto.php?idproy $=20298$

García Pérez, F. F. (2017). Los Principios Didácticos y el Modelo Didáctico Personal. En R. Porlán, (Coord.), Enseñanza universitaria. Cómo mejorarla (pp. 93-104). Ediciones Morata.

García Pérez, F. F. (2019). ¿Es posible educar para la participación ciudadana dentro de la estructura escolar? En S. Claudino et al. (Orgs.), Geografia, Educação e Cidadania (pp. 809-821). ZOE / Centro de Estudos Geográficos, Instituto de Geografia e Ordenamento do Território da Universidade de Lisboa.

García Pérez, F. F. (2021). Algunas dificultades del profesorado de Geografía para educar en ciudadanía. Didacticae, 9, 39-52. https://revistes.ub.edu/index.php/didacticae/article/view/32510

García Pérez, F. F. y De Alba Fernández, N. (2011). Educar en la participación como eje de una educación ciudadana. Reflexiones y experiencias. Didáctica Geográfica, 9, 243-258. https://didacticageografica.agegeografia.es/index.php/didacticageografica/article/view/16

García Pérez, F. F. y Porlán Ariza, R. (16 de febrero de 2000). El Proyecto IRES (Investigación y Renovación Escolar). Biblio $3 W$. Revista Bibliográfica de Geografia y Ciencias Sociales, V(205). http://www.ub.edu/geocrit/b3w-205.htm

García Pérez, F. F., Burgos Sánchez, M. y Guerrero Fernández, A. (2020). La construcción de la ciudadanía en la formación inicial del profesorado de Educación Infantil. Didáctica de las Ciencias Experimentales y Sociales, 39, 4764. https://doi.org/10.7203/dces.39.17121

García Pérez, F. F., De Alba Fernández, N. y Navarro Medina, E. (2015). La formación inicial del profesorado para enseñar ciudadanía. Experiencias en los niveles de grado y de máster. En B. Borghi, F. F. García Pérez y O. Moreno Fernández (Eds.), Novi Cives. Cittadini dall'infanz̧ia in poi (pp. 137-148). Pàtron Editore.

Gasca-Pliego, E. y Olvera-Garcia, J. C. (2011). Construir ciudadanía desde las universidades, responsabilidad social universitaria y desafíos ante el siglo XXI. Convergencia. Revista de Ciencias Sociales, 18(56), 37-58. https://www.redalyc.org/pdf/105/10516855002.pdf

Gomes Dias, A., Gómez Rodríguez, A. E., Santisteban Fernández, A. y Pagès Blanch, J. (2020). Global Citizenship Education and Teacher Education in Spain and Portugal. En D. Schugurensky y Ch. Wolhuter (Eds.), Global Citizenship Education in Teacher Education. Theoretical and Practical Issues (pp. 201214). Routledge.

González Puentes, F. (2013). Dificultades para educar en la participación ciudadana: visión desde el profesorado. En Memorias XIX Congreso Institucional de Investigaciones (No. 3, pp. 215-221). Editorial Universidad El Bosque.

González Puentes, F. (Dir.) (2015). Educación para la cindadania: dificultades y posibilidades de los curriculos y los docentes de la Universidad El Bosque para educar en la participación ciudadana. Proyecto de investigación, Código: PCI-2014-60. Vicerrectoría de investigaciones. Universidad El Bosque. Bogotá.

González Puentes, F., Rodriguez, A., Cortés, E., Becerra, A. y Santafé, C. (2016). Educación para la Ciudadanía: Dificultades y posibilidades de los currículos y los docentes para educar en la participación 
ciudadana. En Memorias XXVI Congreso Institucional de Investigaciones (No. 7, p. 65). Editorial Universidad El Bosque.

González Rivero, B. M. (2019). Retos de la formación ciudadana para la educación superior. Universidady Sociedad, 11(4), 341-349. $\quad$ http://scielo.sld.cu/scielo.php?script=sci arttext\&pid=S2218$\underline{36202019000400341}$

Goren, H. y Yemini, M. (2017). Global citizenship education redefined: A systematic review of empirical studies on global citizenship education. International Journal of Educational Research, 82, 170-183.

Hart, R. (2001). La participación de los niños en el desarrollo sostenible. Ediciones PAU.

Henderson, D. X. y Wright, M. (2015). Getting students to "go out and make a change": Promoting dimensions of global citizenship and social justice in an undergraduate course. Journal of Contemporary Issues in Higher Education, 1, 14-29.

Holguín Villa, L. Y., Penilla González, J. A., Chaverra Rodríguez, L. M. y Arango Zuleta, E. P. (2019). Importancia de la formación ciudadana en el currículo universitario. Revista de Investigaciones UCM, 19(33), 79-90. http://www.revistas.ucm.edu.co/ojs/index.php/revista/article/view/126

Hortas, Mà. J., Dias, A. y De Alba Fernández, N. (2019). Enseñar y aprender didáctica de las ciencias sociales: la formación del profesorado desde una perspectiva sociocrítica. Asociación Universitaria del Profesorado de Didáctica de las Ciencias Sociales, Politécnico de Lisboa y Escola Superior de Educação de Lisboa. Recuperado de http://didactica-ciencias-sociales.org/wp-content/uploads/2019/10/XXXpublicacion-simposio-lisboa-final compressed-min.pdf

Kerr, D. (2000, September). The making of citizenship in the National Curriculum (England): Issues and challenges. Paper presented at the European Conference on Educational Research (Vol. 20, p. 23).

López López, P. (2008). Educación para la ciudadanía: también en la Universidad. El Viejo Topo, 246/247, 95-101. Recuperado de https://eprints.ucm.es/id/eprint/9783/

Lozano Estivalis, M., Traver Martí, J. A. y Segarra Arnau, T. (2018). Responsabilidad Educativa para un Compromiso Ciudadano Crítico. Archivos Analíticos de Politicas Educativas, 26(97), 6 de agosto 2018. https://epaa.asu.edu/ojs/article/view/3688/2107

Magendzo, A. (2004). Formación cindadana. Cooperativa Editorial Magisterio.

Manzano-Arrondo, V. (2011). La universidad comprometida. Hipatia.

Martínez Martín, M. (2006). Formación para la ciudadanía y educación superior. Revista Iberoamericana de Educación, 42, 85-102. https://rieoei.org/historico/documentos/rie42a05.pdf

Martínez-Usarralde, M. J., Lloret-Catalá, C. y Mas-Gil, S. (2017). Responsabilidad Social Universitaria (RSU): Principios para una universidad sostenible, cooperativa y democrática desde el diagnóstico participativo de su alumnado. Archivos Analíticos de Políticas Educativas, 25(75). https://doi.org/10.14507/epaa.25.2769

Molina, S., Miralles, P. y Ortuño, J. (2013). Concepciones de los futuros maestros de Educación Primaria sobre formación cívica y ciudadana. Educatio Siglo XXI, 31(1), 105-126. https://revistas.um.es/educatio/article/view/175361/148441

Morin, E. (2001). Los siete saberes necesarios para la educación del futuro. Paidós.

Moscoso Merchán, F. y Hernández Díaz, A. (2015). La formación pedagógica del docente universitario: un reto del mundo contemporáneo. Revista Cubana de Educación Superior, 34(3), 140-154. http://scielo.sld.cu/scielo.php?script $=$ sci arttextypid=S0257-43142015000300011

Muñoz Zamora, G. (2011). La democracia y la participación en la escuela: ¿Cuánto se ha avanzado desde las normativas para promover la participación escolar? REXE. Revista de estudios y experiencias en educación, 10(19), 107-129. https://www.redalyc.org/articulo.oa?id=243120126006

Nigris, E. (Dir.) (2015). Pedagogia della cittadinanza e formazione degli insegnanti: un'alleanza tra scuola e territorio (Citizenship pedagogy and teacher education: an alliance between school, territory, community). STEP (School, Territory, Environment, Pedagogy). Proyecto de Investigación Erasmus Plus, 2015-1-IT02-KA201-015190. Agenzia Nazionale INDIRE. Italia https://www.formazione.unimib.it/it/ricerca/progetti-ricerca/progettierasmus

Padilla, L. A., Padilla, J. E. y Silva, W. H. (2012). La formación ciudadana en los estudiantes. Revista de Investigaciones UNAD, 11(1), 49-71. https://doi.org/10.22490/25391887.772

Pagès Blanch, J. y Santisteban Fernández, A. (Coords.) (2011). Les qüestions socialment vives i l'ensenyament de les ciències Socials. Servei de Publicacions de la Universitat Autònoma de Barcelona. Col. Documents, 9.

Pineda-Alfonso, J. A. (2015). Educar para la ciudadanía trabajando con temas controvertidos en Educación Secundaria Obligatoria. Revista de Investigación Educativa, 33(2), 353-367. https://doi.org/10.6018/rie.33.2.208441

Pineda-Alfonso, J. A., De Alba-Fernández, N. y Navarro-Medina, E. (eds.) (2018). Handbook of Research on Education for Participative Citizenship and Global Prosperity. IGI Global. 
Ramalho, B. L. y Beltrán, J. (2012). Universidad y sociedad: la pertinencia de educación superior para una ciudadanía plena. Revista Lusófona de Educação, 21, 33-52. https://www.redalyc.org/pdf/349/34924585003.pdf

Rivera de Parada, A. (2013). Cultura politica democrática desde la educación superior. Conocimiento y promoción según docentes universitarios. Ciencia, Cultura y Sociedad, 1(2), 1-13. Recuperado de http://www.redicces.org.sv/ispui/handle/10972/2487

Salazar, L. (2003). La construcción de ciudadanía en educación superior. Docencia Universitaria, 4(2), 31-45. http://saber.ucv.ve/ojs/index.php/rev docu/article/view/4569

Santisteban Fernández, A. (2012). La investigación sobre el desarrollo de la competencia social y ciudadana para una participación crítica. En N. De Alba Fernández, F. F. García Pérez y A. Santisteban Fernández (Eds.), Educarpara la participación ciudadana en la enseñanza de las ciencias sociales (vol. I, pp. 277-286). Díada Editora.

Salinas Valdés, J., Oller Freixa, M. y Muñoz Labraña, C. (2016). Representaciones sociales de la participación ciudadana en docentes de ciencias sociales: perspectivas para la nueva asignatura de formación ciudadana en Chile. Foro Educacional, 27, 141-161. http://ediciones.ucsh.cl/index.php/ForoEducacional/article/view/801/747

Soler, P., Pallisera, M., Planas, A., Fullana, J. y Vilà, M. (2012). La participación de los estudiantes en la universidad: dificultades percibidas y propuestas de mejora. Revista de Educación, 358, 542-562. http://www.revistaeducacion.educacion.es/re358/re358 25.pdf

UNESCO (1998). Declaración mundial sobre la educación superior en el siglo XXI: visión y acción. UNESCO. Recuperado de https://deportes.utem.cl/wp-content/uploads/2016/11/02-Declaraci\%C3\%B3nMundial-sobre-la-Educaci\%C3\%B3n-Superior-UNESCO.pdf

UNESCO (8 de julio de 2009). Conferencia Mundial sobre la Educación Superior - 2009: La nueva dinámica de la educación superior y la investigación para el cambio social y el desarrollo. [Comunicado]. UNESCO. Recuperado de

https://pep.unc.edu.ar/wpcontent/uploads/sites/46/2017/04/Declaracion conferencia Mundial de Educacion Superior 20 $\underline{09 . p d f}$

UNESCO (2016). Educación para la ciudadanía mundial. Preparar a los educandos para los retos del siglo XXI. Recuperado de http://www.fundacioncives.org/rec/recursos/educacion-para-la-ciudadania-mundialpreparar-a-los-educandos-para-los-retos-del-siglo-xxi.html

Vallaeys, F., y Carrizo, L. (2006). Hacia la construcción de indicadores de responsabilidad social universitaria. [Documento]. Recuperado http://www.centroetica.uct.cl/documentos/archivos/PDF/H1\%2002.pdf

Velásquez, F. (2003). La participación ciudadana en Bogotá: Mirando el presente, pensando el futuro. Alcaldía Mayor de Bogotá. 